\title{
Medial Lemniscus Tract Lesion After High Voltage Electrical Injury: A Case Report
}

\author{
Chull-Hyun Cho, MD ${ }^{1}$, Dong Gyu Lee, $\mathrm{MD}^{2}$ \\ ${ }^{1}$ Department of Orthopedic Surgery, Keimyung University School of Medicine, Daegu; ${ }^{2}$ Department of Physical Medicine and \\ Rehabilitation, Yeungnam University College of Medicine, Daegu, Korea
}

\begin{abstract}
We present the case of a 33-year-old man who experienced a 10,000-V electrical shock when working with electrical wiring. He suffered third-degree burns on his scalp at the right occiput (entry wound) and on his left arm (exit would), and a second-degree burn on his left foot (exit wound). He presented with severe spasticity of both lower extremities, motor weakness with a Medical Research Council grade of 3, and sensory impairments below thoracic level 11 that included an inability to sense light touch and defects in proprioception. Initial magnetic resonance imaging (MRI) scans of his spine and brain showed no definite abnormalities. However, tractography obtained by diffusion tensor imaging of the brain showed absence of the right medial lemniscus tract. A cervical MRI scan 1 month later showed spinal cord swelling from cervical 1-5 levels, and signal changes in the lateral and posterior white matter in the axial view. After 6 months of rehabilitation, he recovered almost normal degree of motor function in his lower extremities and disappearance of spasticity. However, since the sensory impairments persisted, especially defects in proprioception, he was unable to walk independently.
\end{abstract}

Keywords Electric injuries, Burns, Medial lemniscus, Diffusion tensor imaging

\section{INTRODUCTION}

High voltage electrical injury results in various types of damage to soft tissue, muscles, bones, and the nervous system [1]. Depending on the degree of damage, injury to the central nervous system is associated with a wide range of prognosis, from permanent impairment to full recovery [2]. Therefore, for patients with central nervous system damage, it is important to be evaluated soon after the injury occurs. Accurate evaluation of the damage enables clinicians to devise a proper strategy for rehabilitation. However, assessing the degree of nervous system damage in patients who suffered high-voltage injuries is challenging. Clinical neurologic deficits sometimes do not correspond with radiologic findings in the early stages [3]. Delayed progressive neurologic deficits can occur in some cases, which makes determination of the severity of central nervous system damage by radiologic evaluation difficult [4]. Moreover, separation of myelinated axons and injury to the cytoskeleton can be seen on

Received March 2, 2016; Accepted June 13, 2016

Corresponding author: Dong Gyu Lee

Department of Physical Medicine and Rehabilitation, Yeungnam University College of Medicine, 170 Hyeonchung-ro, Nam-gu, Daegu 42415, Korea. Tel: +82-53-620-3270, Fax: +82-53-620-3270, E-mail: hikkali@hanmail.net

ORCID: Chul-Hyun Cho (http://orcid.org/0000-0003-0252-8741); Dong Gyu Lee (http://orcid.org/0000-0002-4787-4448).

(c) This is an open-access article distributed under the terms of the Creative Commons Attribution Non-Commercial License (http://creativecommons.org/ licenses/by-nc/4.0) which permits unrestricted noncommercial use, distribution, and reproduction in any medium, provided the original work is properly cited. Copyright ( 2017 by Korean Academy of Rehabilitation Medicine 
an electron micrograph by electrical stimulation without heating [5]. Diffusion tensor tractography (DTT) has been used to investigate the structural changes associated with white matter pathology. Hence, we used DTT to evaluate changes in axonal integrity within the white matter after a high-voltage electrical injury.

We report a case of damage to the medial lemniscus tract in the brain after high-voltage electrical injury, assessed by DTT.

\section{CASE REPORT}

While working on electrical wiring, a 33-year-old male fell from a height of $1.5 \mathrm{~m}$ after receiving a 10,000-V electrical shock from a high-voltage power line. The patient had third-degree burns on his left arm and right scalp (Fig. 1). Because of the burn and the presence of compartment syndrome, the left arm was amputated below the elbow; later, due to the development of necrosis on the stump, an additional amputation above the elbow was done. To treat the right scalp injury, debridement of dehiscence and skull necrosis was performed, followed by flap surgery. The patient had neurologic deficits manifesting as spasticity, motor weakness, and sensory impairment below thoracic level 11. According to the International Standards for Neurological Classification of Spinal Cord Injury (ISNCSCI) scoring system, the total

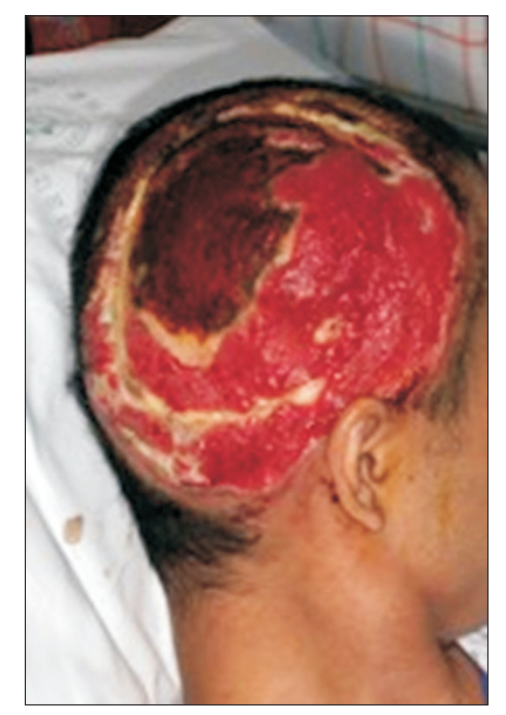

Fig. 1. A third-degree burn on the right scalp of the patient, and skull necrosis. lower extremity motor score (LEMS) was 30 points. Spasticity of the lower extremities from the hips to the ankles was grade 2 on the Modified Ashworth Scale (MAS). There were severe deficits in sensitivity to light touch and proprioception in the area below the knee. After surgery, the patient was transferred to the rehabilitation department for treatment of paraplegia and the left above elbow amputation. The degree of spasticity gradually decreased; 2 months after the injury, only spasticity of MAS grade 1 remained in the left ankle plantar flexors. Motor tone and performance in the lower extremities improved to a LEMS of 46 points; 5 months after the injury, but the patient was unable to stand independently due to continued impairments in proprioception.

Cervical spine magnetic resonance imaging (MRI) and brain MRI scans done 1 week after the injury did not show any specific abnormalities, except a signal change in the right temporal lobe (Fig. 2A, 2C). Since the MRI findings did not match symptoms of the patient, DTT was performed to investigate for white matter abnormalities. No specific abnormalities in the corticospinal tract (CST) were seen, but both medial lemniscus (ML) tracts were imperceptible (Fig. 3A). Thirty days after the injury, an additional cervical spine MRI was conducted, and swelling and signal changes were observed at cervical levels 1 to 5 (Fig. 2B). Lateral and posterior white column signal change was especially prominent on a T2-weighted axial view (Fig. 2D). Four months after the injury, follow-up diffusion tensor imaging was conducted, in which the left ML was still unseen (Fig. 3B, 3C). Sensory impairment persisted 18 months after the injury. Till that time, the patient was unable to stand or walk independently.

\section{Diffusion tensor imaging and tractography}

The diffusion tensor imaging (DTI) was acquired using a sensitivity-encoding head coil on a 3T MR scanner (Signa EXCITE; GE Healthcare, Waukesha, WI, USA). For each of the 26 non-collinear diffusion-sensitizing gradients, a diffusion-weighted echo-planar imaging sequence was performed with the following parameters: TR/TE/ NEX, $10000 \mathrm{~ms} / 95.9 \mathrm{~ms} / 2.0$; slice thickness, $2.6 \mathrm{~mm}$; b, $1000 \mathrm{~s} / \mathrm{mm}^{2}$; matrix $128 \times 128$; FOV, $250 \times 250 \mathrm{~mm}$. A probabilistic tractography method based on a multi-fiber model was used for fiber tracking, and applied in the current study utilizing tractography routines implemented in FMRIB Diffusion (0.5 mm step lengths, 5000 stream- 

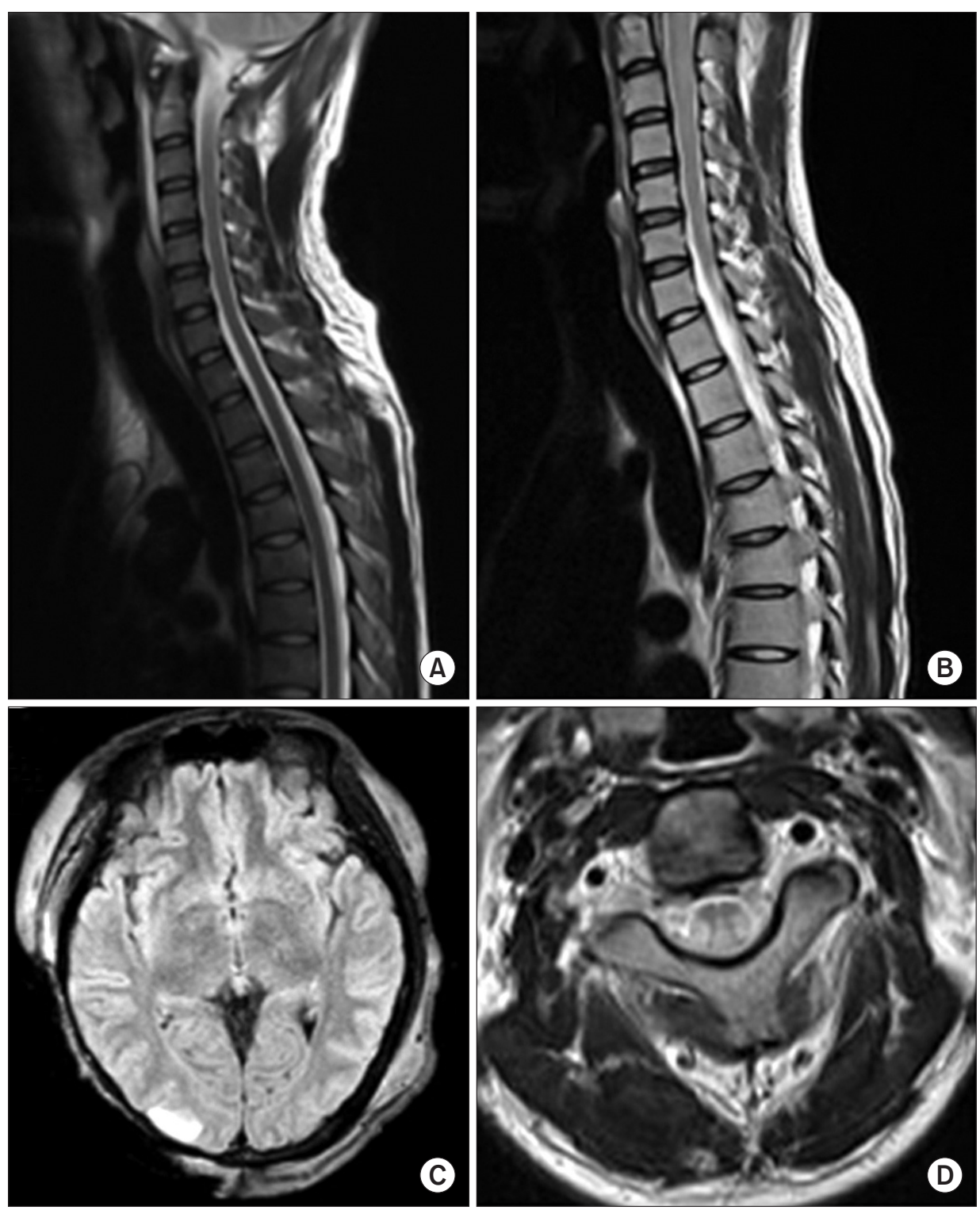

Fig. 2. (A, C) A cervical spinal magnetic resonance imaging (MRI) taken 1 week after the injury reveals no specific findings, but a brain MRI shows signal changes in the right temporal lobe. (B) A cervical spine T2-weighted MRI, taken 30 days after the injury, shows swelling and signal changes from $\mathrm{C} 1$ to C5. (D) An axial T2weighted image at the $\mathrm{C} 2-3$ level shows damage to the lateral and posterior column.

line samples, curvature thresholds $1 / 4$ 0.2) [6]. The region of interest (ROI) was determined based on previously reported anatomical locations [7]. The seed ROI of CST was placed on the portion of the pontomedullary junction, and target ROI was the portion of the anterior midpons and primary motor cortex. The seed ROI of ML was located medio-posteriorly to the medullary pyramid, and two target ROIs were defined in the ventral posterolateral nucleus (VPL) of the thalamus and the primary somatosensory cortex. Eddy current-induced image distortions were removed using affine multi-scale two-dimensional registration, available from the Oxford Centre for Functional Magnetic Resonance Imaging of the Brain (FMRIB) software library (FSL; http://www.fmrib.ox.ac.uk/fsl).

\section{DISCUSSION}

Electrical injury causes damage to soft tissue, muscles, bones, and the nervous system. Among these regions, damage to the central nervous system is one of the most common complications of high-voltage electrical accidents [8]. Delayed progressive neurologic deficits are frequently seen, which are caused by the formation of a thrombus in an injured small vessel, or diffuse degenerative changes to vessel walls [9]. A disconnect between neurological damage and radiologic findings in the early stages of injury is often reported, and in such cases, radiologic findings alone are unable to accurately assess the extent of neural damage or determine the prognosis. 

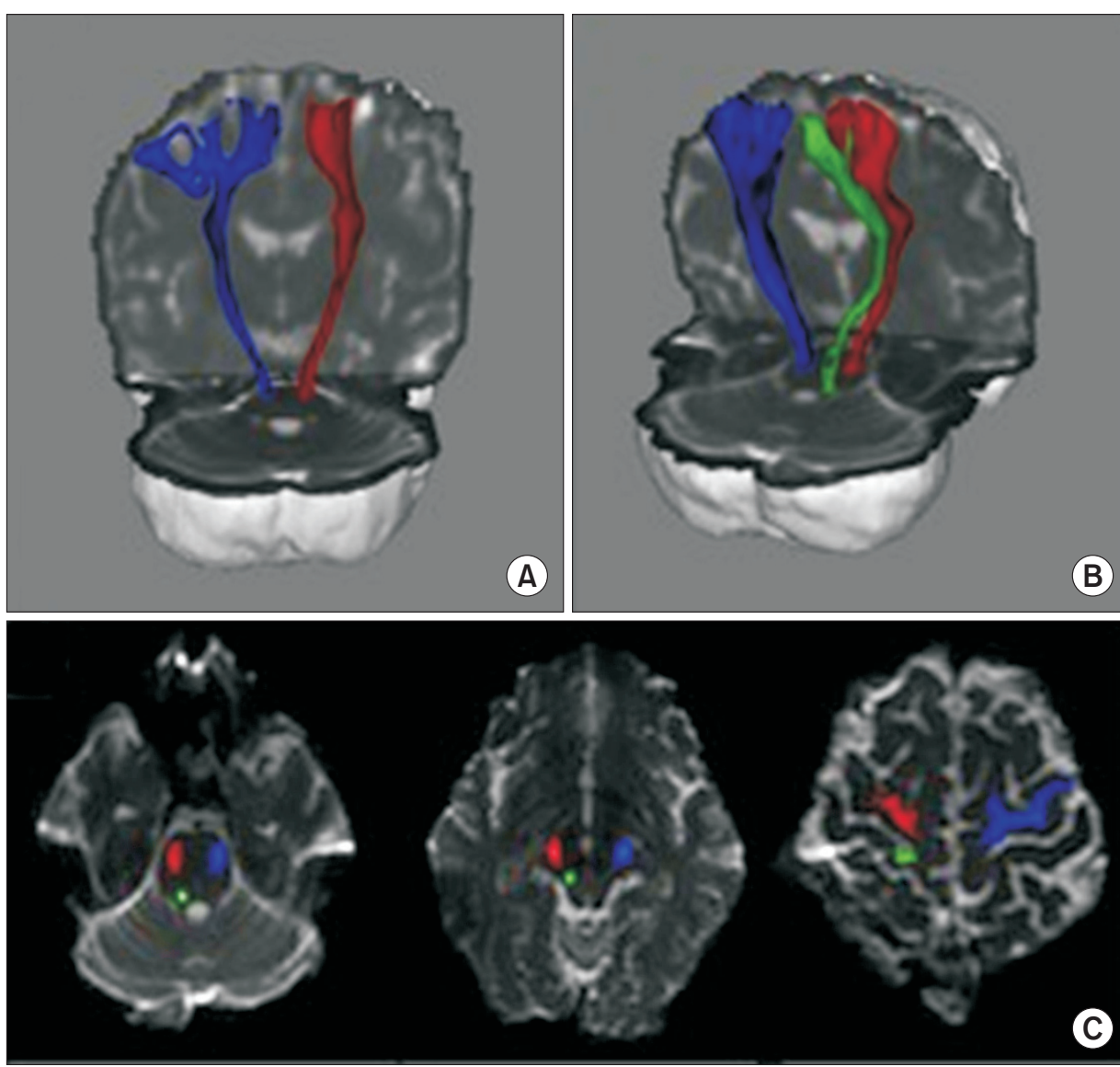

Fig. 3. Tractography of the corticospinal tract (CST) and medial lemniscus tract (ML). (A) The right CST (red) and left CST (blue) are well-connected on each anatomical level from the brain stem to the cortex. (A, B) Although the ML (green) is seen in the right hemisphere, it cannot be viewed in the left hemisphere. Both CST and right ML are properly located on the anatomical locations from cerebral cortex to pons level (C).

Case reports have shown various types of damage to the central nervous system and associated symptoms, based on the location of the entrance and exit wounds. Reports contain clinical, histologic and radiologic findings, which commonly include deficits in sensation and proprioception. From a clinical perspective, some patients report difficulty in functional activity due to loss of the vibration sense and proprioception in both legs. Seo et al. [10] reported damage to the posterior column of the spinal cord, as a histologic finding in animal tests using a highvoltage electrical injury model. In that study, electrical injury produced cavitation and rupture in the middle of the posterior column. Injury to the posterior white matter results in prominent proprioceptive deficits in the lower extremities, since the posterior column is organized somatotopically. Our patient also showed signal changes in the lateral and posterior column in the axial view of the delayed cervical spine MRI. Hence, central ML and cervical posterior column injury may synergistically produce proprioceptive impairment below thoracic level 11.

Electrical injury decreases the number of neural cells in the ventral horn [10]. We therefore presume that injuries to cells in the ventral horn and lateral column gave rise to motor weakness in our patient. The motor function after the injury gradually improved to 46 points on the LEMS. However, proprioception remained impaired in both lower extremities, so that independent standing was impossible.

Diffusion tensor image is advantageous in that it allows to study the structural continuity of white matter neural tract. Tract tracking in this case was designed to determine the degree of brain injury causing impairments in motor performance and sensory function by analyzing CST and ML tract. The CST was seen to be relatively spared, but both ML tracts were absent. Although the follow-up tractography showed the left ML tract to be relatively intact, anatomical lesions were found in the ML tract in both hemispheres on DTI, and damage to the posterior column was evident on delayed T2-weighted cervical spine MRI, which explained the lasting defects in proprioception.

The CST did not reveal any specific damage on trac- 
tography, but the ML tract was severely damaged. Although it is difficult to explain clearly, we propose two hypotheses. First, unlike CST, medial lemniscus pathway within the brain has several synapses. Ascending axons from posterior column make contact with the gracile and cuneate nucleus in the lower medulla, distributing their axons to the VPL of the thalamus on the contralateral side. Subsequently, neurons starting in the VPL in the thalamus travel up to the cerebral cortex. Thus, these multiple synapses within the brain possibly results in the ML tract being vulnerable to injury of high-voltage electrical insult. Second, a previous study reported that highvoltage electricity causes damage to myelinated tissue [5]. Thus, although the extent of damage to myelinated axons was not severe enough to produce abnormal findings on T1- and T2-weighted images, it could have been severe enough to affect anisotropy, which would be evident on tractography.

There are few reports of high-voltage electrical injury affecting the spinal cord and brain. The mechanisms of damage to the central nervous system after high-voltage electrical injury are therefore not fully elucidated. Our case demonstrates that the sensory tracts of the central nervous system are vulnerable to high-voltage electrical injury. DTT is a useful tool to determine the extent of brain injury undetectable by routine MRI scans taken soon after injury.

\section{CONFLICT OF INTEREST}

No potential conflict of interest relevant to this article was reported.

\section{REFERENCES}

1. Arnoldo BD, Purdue GF, Kowalske K, Helm PA, Burris
A, Hunt JL. Electrical injuries: a 20-year review. J Burn Care Rehabil 2004;25:479-84.

2. Ratnayake B, Emmanuel ER, Walker CC. Neurological sequelae following a high voltage electrical burn. Burns 1996;22:574-7.

3. Ko SH, Chun W, Kim HC. Delayed spinal cord injury following electrical burns: a 7-year experience. Burns 2004;30:691-5.

4. Clouston PD, Sharpe D. Rapid recovery after delayed myelopathy from electrical burns. J Neurol Neurosurg Psychiatry 1989;52:1308.

5. Tun K, Cemil B, Gurcay AG, Kaptanoglu E, Sargon MF, Tekdemir I, et al. Ultrastructural evaluation of Pulsed Radiofrequency and Conventional Radiofrequency lesions in rat sciatic nerve. Surg Neurol 2009;72:496500; discussion 501.

6. Smith SM, Jenkinson M, Woolrich MW, Beckmann $\mathrm{CF}$, Behrens TE, Johansen-Berg $\mathrm{H}$, et al. Advances in functional and structural MR image analysis and implementation as FSL. Neuroimage 2004;23 Suppl 1:S208-19.

7. Jang SH, Kwon HG. Anatomical location of the medial lemniscus and spinothalamic tract at the pons in the human brain: a diffusion tensor tractography study. Somatosens Mot Res 2013;30:206-9.

8. Varghese G, Mani MM, Redford JB. Spinal cord injuries following electrical accidents. Paraplegia 1986;24:159-66.

9. Erkin G, Akinbingol M, Uysal H, Keles I, Aybay C, Ozel S. Delayed cervical spinal cord injury after high voltage electrical injury: a case report. J Burn Care Res 2007;28:905-8.

10. Seo CH, Jeong JH, Lee DH, Kang TC, Jin ES, Lee DH, et al. Radiological and pathological evaluation of the spinal cord in a rat model of electrical injury-induced myelopathy. Burns 2012;38:1066-71. 